\title{
Early Time Bolometric Light Curves of Type-II Supernovae Observed by Swift
}

\author{
T. A. Pritchard ${ }^{1}$ and P. W. A. Roming ${ }^{2,1}$ \\ ${ }^{1}$ Dept. of Astronomy \& Astrophysics, The Pennsylvania State University, \\ 525 Davey Lab, University Park, PA 16802, USA \\ email: tapritchard@astro.psu.edu \\ ${ }^{2}$ Southwest Research Institute, Department of Space Science \\ 6220 Culebra Rd, San Antonio, Texas, 78238 USA \\ email: proming@swri.edu
}

\begin{abstract}
We present early time ( $\sim 0-50$ days) bolometric light curves of UV-bright Core Collapse Supernovae observed with the Swift UV/Optical Telescope. We also generate pseudobolometric light curves from Swift UV and optical data and examine these by subtype as well as the observed and interpolated UV and IR flux contributions by epoch and bolometric corrections at early times from UV data.
\end{abstract}

Keywords. supernovae:general, ultraviolet: general, catalogs

\section{Introduction}

We present early time UV and optical observations of Core-Collapse SNe (CCSNe) obtained by Swift since its launch in 2005. UV observations of these objects at early times are rare, and for UV-bright objects (II, IIP, IIn, IIL SNe) the UV may account for upwards of $80 \%$ of the total luminosity during this early portion. Due to the paucity of UV instruments available this large fraction of a SN's light often goes unaccounted for in the first several weeks of a SN. To help rectify this situation we present a collection of observations by the Swift satellite in the UV and optical bands. We generate pseudobolometric light curves from this data, and using blackbody fits to approximate the UV and IR tails outside of Swift coverage compute a bolometric correction to arrive at a bolometric light curve. We are working to incorporate observations from other telescopes currently so that we may increase the observed coverage of these SNe and increase the quality of our bolometric light curves.

\section{Overview}

We use synthetic photometry from reddened model blackbodies fit to the Swift light curve data, and UVOT count rate to flux conversions interpolated from data in Appendix A of Brown et al. 2010 using our best fit blackbody temperature, to generate a pseudobolometric light curve for each CCSN. At each epoch we then use the integrated flux of the best-fit reddened model blackbody at our unobserved wavelengths to calculate UV $(<1600$ Angstrom) and IR (>6000 Angstrom) corrections. These correction factors are then added to the pseudo-bolometric light curve to arrive at a bolometric light curve. Due to the nature of the CCSNe spectra, and the Swift filter selection we find that this works best for SNe with a thick hydrogen shell as these SNe are both typically UV-bright at early times as well as the SNe spectra more closely resembling our blackbody model. 

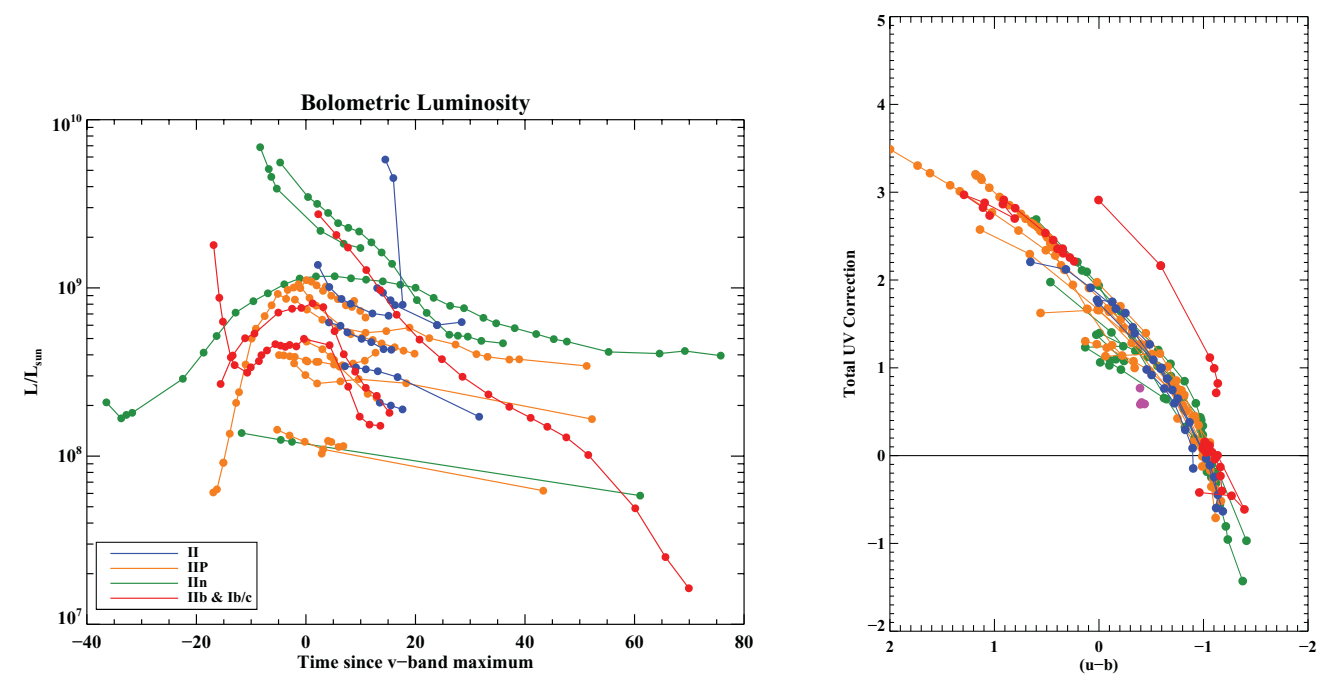

Figure 1. Left: UVOT Bolometric lightcurves from the Swift sample centered around the time of maximum light in the visual band. Right: Correction factor in magnitudes as a function of CCSNe color

\section{Implications}

Using the Swift only data we find a maximum unobserved UV correction of only $\sim 25 \%$ of the total flux. This is a factor of $>3$ better than can be done with ground based optical and IR data only at similar epochs, as seen in Bersten \& Hamuy 2009. This allows us to calculate an accurate distance-independent UV correction based on optical colors as visible in Figure 1) (Right). We perform this calculation in a similar manner as normally done for a bolometric correction; but we only include Swifts observed UV flux (filters $u$, uvw1, uvm2, and uvw2 filters) plus the calculated UV correction factor. We find that all CCSNe subtypes behave similarly, however $u-b$ color appears to be a much stronger indicator than $b-v$. This is expected since $u$-band traces the UV-flux much more closely than $b$.

In Figure 1 (Left) we plot our sample's bolometric light curves. These UV-bright CCSNe reach maximum light very rapidly, which is seen in our sample in both the lack of a clear UV-rise in most SNe UV light curves as well as the few clear peaks we see in the bolometric light curves. While our IR-correction at early times is quite small, as low as $5 \%$ for some CCSNe, after $\sim 20$ days post $v$-band maximum this has grown to be significant. At the end of Swift observations, due to the SNe spectrum cooling, we have found this correction to be as high as $80 \%$ of the total SNe flux. Integration of optical and IR ground based observations red-ward of the UVOT bandpass are underway, and are necessary to enhance the quality of the bolometric light curves at these times. Further observations of very early supernovae to observe the UV rise will continue to improve these early time bolometric light curves as well.

\section{References}

Bersten, Melina C. \& Hamuy, Mario 2009, ApJ, 701, 200

Brown, Peter J., et al. 2010, ApJ 721, 1608 